\title{
Host-Microbial Gut Interactions and Mushroom Nutrition
}

\author{
Victoria Bell $^{1}$, Jorge Ferrão ${ }^{2}$, Eusébio Chaquisse ${ }^{3}$, Tito Fernandes ${ }^{4, *}$ \\ ${ }^{1}$ Faculty of Pharmacy, Coimbra University, Pólo das Ciências da Saúde, 3000-548 Coimbra, Portugal \\ ${ }^{2}$ The Vice-Chancellor’s Office, Universidade Pedagógica, Rua João Carlos Raposo Beirão 135, Maputo, Moçambique \\ ${ }^{3}$ National Institute of Health, Ministry of Health, Av 24 Julho, Maputo, Mozambique \\ ${ }^{4}$ Faculty of Veterinary Medicine, Lisbon University, 1300-477 Lisboa, Portugal \\ *Corresponding author: profcattitofernandes@gmail.com
}

Received August 05, 2018; Revised September 12, 2018; Accepted September 20, 2018

\begin{abstract}
There is a tremendous complexity of the human gut microbiota in both health and disease states and a healthy microbiota consists of an inter-dependent network of microbes rather than a particular bacterial genera. The microbiota of the gastrointestinal tract is a symbiotic partner of the host as it is crucial for maintaining homeostasis and multiple components of the host immune system. Numerous host factors influence the composition of the microbiota early in life including diet, hygiene, environmental contacts, antibiotic use, and breastfeeding. Although the content of any diet can effect bacterial composition, it cannot be suggested that diet alone is responsible for the diversity of the microbiota or its variation among individuals. The intestinal physical-chemical barrier forms part of the intestinal immune system and plays a critical role in determining the composition of the microbiota. There are multiple recognised clinical uses of mushrooms due to their content in $\beta$-glucans, important antioxidant and cytoprotective enzymes, secondary metabolites and still other unknown factors. Mushroom $\beta$-glucans have been proposed to act as "biological response modifiers" based on their effects on the immune system, enhancing the body's own use of macrophages and T-lymphocytes, rather than directly attacking any tumours, controlling oxidative stress and inflammation.
\end{abstract}

Keywords: microbiota, mushroom, $\beta$-glucans, immunity, gut

Cite This Article: Victoria Bell, Jorge Ferrão, Eusébio Chaquisse, and Tito Fernandes, "Host-Microbial Gut Interactions and Mushroom Nutrition." Journal of Food and Nutrition Research, vol. 6, no. 9 (2018): 576-583. doi: 10.12691/jfnr-6-9-6.

\section{Introduction}

Humans live in association with free-living or colonial protists microrganisms, bacteria, viruses, fungi, archaea and there is also growing evidence suggesting that helminths and protozoa may significantly interact with human gut bacteria $[1,2]$. The 20th century saw incredible advances in our understanding of host-microbe interactions, with identification of numerous disease-associated organisms and elucidation of pathogenic mechanisms. However, the vast majority of this work was directed at purging the human body of pathogenic organisms. While all of these efforts have dramatically reduced morbidity and mortality related to infections, they may also have inadvertently rid us of needed immunomodulatory molecules [3].

Researchers have spent more than 100 years studying pathogens with the aim of their eventual elimination, with little regard for what happens in their absence; it is now clear that many "pathogens" more commonly exist as commensal organisms. By a variety of measures, the species Homo sapiens is more microbial than human since research has shown that the human body is actually composed of more bacteria than body cells. Collectively, these trillions of bacteria are called the microbiome. Microorganisms comprise only a small, albeit significant, percentage of the body weight (between 900 and $2300 \mathrm{~g}$ of live bacteria). However, in terms of cell numbers, we are about $10 \%$ human and $90 \%$ bacterial [4]. In the future, we need to expand our understanding on the parasitism, commensalism and mutualism among the microbiota and of how pathogenic organisms may in reality be vital to our well-being [5].

Our digestive microbiota is actually directly linked to our brains which is explained explained by a network of neurons lining our guts that is so extensive some scientists have nicknamed it our "second brain" [6]. The gut digestive system has its own nerve system called the enteric nervous system while there are more neurons (nerve cells) in our enteric nervous system than in our brain [7]. A deeper understanding of this mass of neural tissue, filled with important neurotransmitters, is revealing that it does much more than merely handle digestion or inflict the occasional nervous problem but may determine our mental state and play key roles in well-being and certain diseases.

Anaerobic bacteria are prevalent among the bacterial populations of the human body, particularly on mucous 
membrane surfaces. The major sites with a rich anaerobic normal microflora are the mouth, the gastrointestinal tract and the female genital tract. Almost immediately after a human being is born, so too is a new microbial ecosystem, one that resides in that person's gastrointestinal tract. The oral cavity harbours more than 300 different bacterial species. Indeed, the cells in our gut outnumber the cells in the rest of your body by more than ten to one.

The sources of the microbes that make up the ecosystem, how and why it varies from one person to another, and how the composition of this ecosystem influences human digestion, physiology, metabolism, development, and diseases are still poorly understood. About 500 different bacterial species are recovered in the lower intestine. The most common anaerobic microorganisms are bifidobacteria, lactobacilli and bacteroides. Two genera, Prevotella and Bacteroides, were shown to have reciprocal patterns of abundance, while little is known about variation across the world. Almost immediately after a human being is born, so too is a new microbial ecosystem, one that resides in that person's gastrointestinal tract.

Research from the 70's on the rumen and hind gut pioneered studies on the adhesion process of the predominant cellulolytic bacteria Bacteroides (Fibrobacter) succinogenes, Prevotella and Ruminococcus (flavefaciens and albus) which can be divided into four phases: 1) transport to the substrate; 2) nonspecific adhesion of bacteria to unprotected sites of the substrate; 3) specific adhesion via adhesins; 4) proliferation of the attached bacteria on potentially digestible tissues of the substrate [8].

Long term diets strongly affects human health, partly by modulating gut microbiome composition particularly protein and animal fat (Bacteroides) versus carbohydrates (Prevotella) [9]. These anaerobes enhance health of the human host by helping to produce and catabolize complex molecules and the dense resident microbial community in the gut, is essential for many host physiological processes that include enhancement of the intestinal epithelial barrier, development of the immune system, and nutrient acquisition. In the healthy gut, the resident bacteria occupy intestinal colonization niches. A major function of the commensal microbiota is the proliferation and colonization of incoming enteric pathogens as well as opportunistic pathobionts through multiple mechanisms $[10,11]$.

\section{Interactions between Fungi, Bacteria and Gut Wall}

The interactions between bacteria and fungi are of central importance to numerous biological questions in agriculture, forestry, environmental science, food production, and medicine [12]. There are several reports of bacterial-fungal interactions which are important in nature but whose significance remains to be established. Fungi and bacteria often form interdependent consortia relying on a common substrate harbouring characteristics quite distinct from those of their single components [13].

Active hemi/cellulose digestion does not occur directly in humans for lack of specific enzymes, and it involves adherence of cells to the fibres via a glycoprotein glycocalyx, which protects cells from protozoal grazing and cellulolytic enzymes from degradation by bacterial proteases while it retains-at least temporarily-the cellodextrin products for use by the cellulolytic bacteria in the hind gut. There are many interactions between luminal nutrients and gut microbiota metabolites with the gut sensory receptors, and the key communications between endocrine, neuronal and immune systems.

A key factor in determining gut microbiota composition is diet, namely linked to long-term diet composition, suggesting that dietary patterns are associated with distinct combinations of bacteria in the intestine, also called enterotypes. Although already demonstrated the effects of protein and fats on Bacteroides and fibre on Prevotella, it is currently unknown which food constituents specifically promote growth and functionality of beneficial bacteria in the intestine [14].

We share our bodies with a diverse set of microorganisms, known collectively as the human microbiome or microbiota. The relative contributions of genetics, environment, and lifestyle factors, to the composition of human gut microbiota is far from fully understood. The mammalian mucosal gastrointestinal tract is a complex ecological environment that contains a heterogeneous population of $>1014$ microorganisms that belong to $\sim 1,000$ different species [15]. Moreover, the microbiome and host emerged as a unity along evolution by a process of integration [16].

Through their own enzymes gut microbiota breaks down indigestible compounds, such as hemicelluloses, and help preventing the growth of undesirable bacteria. Bacteria breaks down complex $\beta$-glucans ingested, producing short-chain volatile fatty acids-VFA (acetic, propionic, butyric). These VFA are major by-product of anaerobic metabolism and main sources of energy in ruminants, rabbits and horses, while in humans they may increase insulin production from the pancreas, alter glycogen breakdown by the liver and therefore inhibit hepatic glucose production by acting directly on the liver as well as indirectly through effects on adipose tissue, pancreas, and brain.

VFA and several other metabolites behave as biologically active molecules that interact with physiological pathways of the host, organ development, metabolism and immunoregulation. The immune system needs to adapt and tolerate tolerate some microorganisms, while retaining the ability breakdown other microorganisms that cause harm. Microbial products activate interrelated immune, endocrine and central nervous system pathways and, if failed, may increase the risk of autoimmune diseases in which the body mistakenly attacks its own cells and tissues. In addition to priming immunoregulatory circuits, exposure to microbial biodiversity provides crucial data that builds up the antigenic repertoire and activation of the immune system [17].

Good health may require continuous interference between the host and the microbiota in a symbiotic relationship while intestinal lamina propria cells cooperate to maintain physiological homeostasis. The successful establishment of an association between bacteria and fungi has profound consequences for both organisms. The effects of fungal interactions on bacterial 
physiology (and vice versa) can be assessed by using global techniques such as proteomics and transcriptomics [18].

Studies of the human microbiome [19] have revealed that even healthy individuals differ remarkably in the microbes depending on dietary habits and patterns (e.g. vegetable rich diets, in particular containing fibre, vs. diets rich in animal products, mainly meat) lead to significant compositional and functional differences in the gut microbiota. Besides diet, host genetics, habitats, type of birth (e.g. caesarean or natural delivery) may explain the wide diversity.

Humans are colonized by multitudes of commensal organisms representing members of five of the six kingdoms of life; however, our gastrointestinal tract provides residence to both beneficial and potentially pathogenic microorganisms. Imbalances in the composition of the gut microbiota, known as dysbiosis, is associated with the pathogenesis of both intestinal and extra-intestinal disorders. Intestinal disorders include inflammatory bowel disease, coeliac disease, colorectal cancer and irritable bowel syndrome, while non-intestinal disorders include asthma, allergy, the many components of metabolic syndrome and cardiovascular disease [20]. The mutualistic relationship between the colonic microbiota, their metabolic products, and the host immune system may lead to disease development [21].

Most people do not realize that 80 percent of human immune system lies in the gastrointestinal tract and 20 percent in the back of our mouth (the tonsils which serve as the first line of defence in the immune system) enhancing the fact that an optimal health starts in your gastrointestinal tract. This system comprises an immunological network termed the gut-associated lymphoid tissue (GALT) that consists of unique arrangements of B cells, $\mathrm{T}$ cells and phagocytes which sample luminal antigens through specialized epithelia. Intestinal microbial dysbiosis has also been observed in extra-intestinal diseases and in particular those that may impact on the 'gut-brain-axis' to affect the CNS and behaviour and cognitive function [22] and dietary interventions may have the potential to modulate psychiatric symptoms associated with gut-brain axis dysfunction [23] (Figure 1).

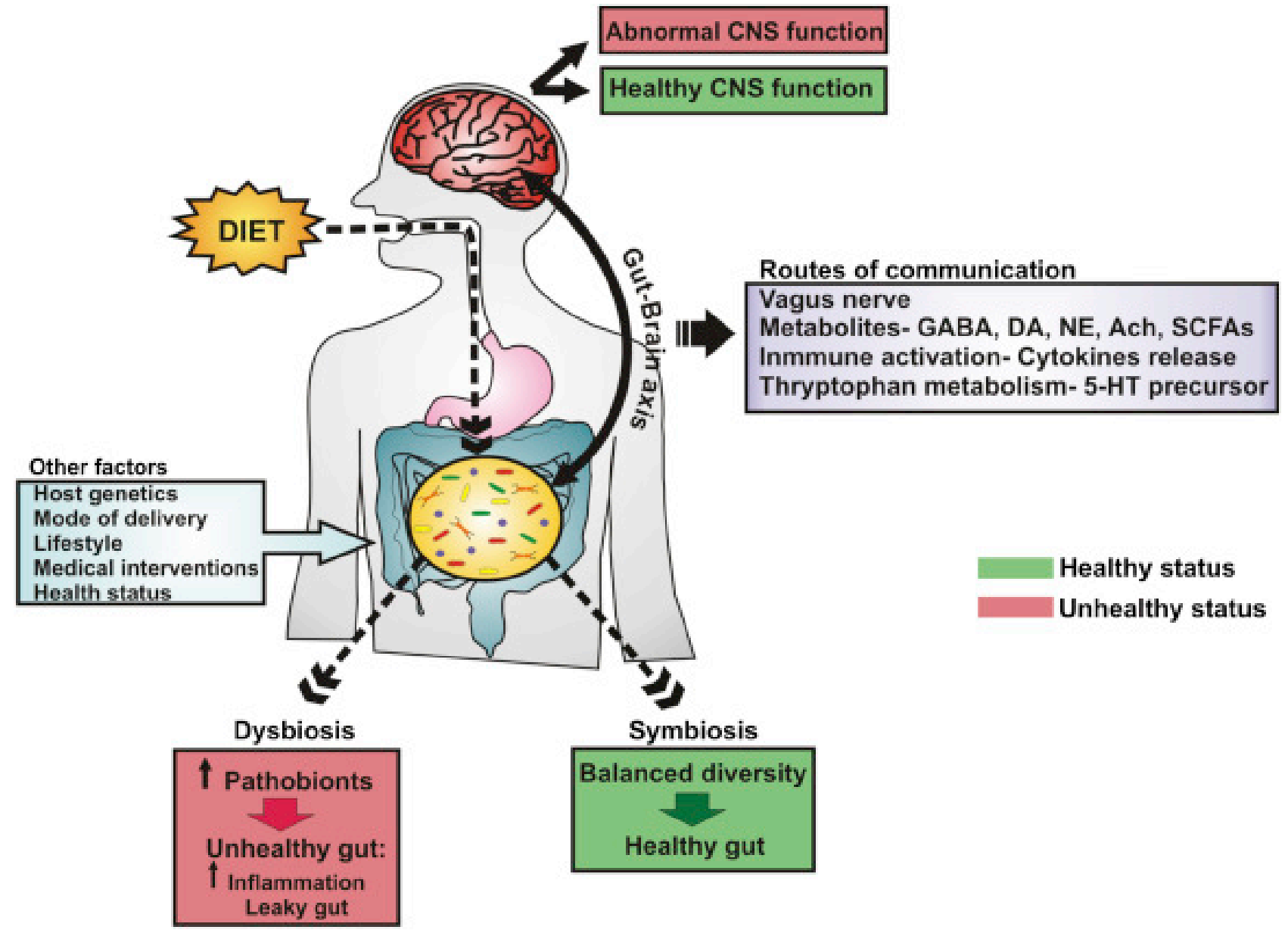

Figure 1. Impact of diet on the gut microbiota and routes of communication involved in the gut-brain axis. Diet is one of the most crucial factors in the development of the human gut microbiota. Different dietary patterns can change the gut microbiota composition by keeping a balanced diversity of the gut microbiota (symbiosis) or causing a state of dysbiosis which is characterized by an overgrowth of potentially pathological organisms (pathobionts). A state of dysbiosis leads to an increased inflammation and leaky gut. Many mechanisms have shown to be involved in this bidirectional pathway between the gut microbiota and brain including vagus nerve signalling, immune activation, tryptophan metabolism and production of microbial metabolites and neurometabolites. Many of these bacterial metabolites significantly impact neurological function, therefore there is potential for dietary interventions that increase bacterial metabolism and promote growth of beneficial bacteria, to beneficially modulate the gut-brain axis and modulate CNS function. Abbreviations: GABA, gamma-aminobutyric acid; DA, dopamine; NE, norepinephrine; Ach, acetylcholine; SCFAs, short-chain fatty acids; 5-HT,serotonine; CNS, central nervous system [23] 


\section{Multiple Uses of Mushrooms}

There are multiple recognised clinical uses of mushrooms due to their content in $\beta$-glucans. Mushroom $\beta$-glucans, by boosting or modulating the immune system are used to lower cholesterol levels and balance fasting blood glycemia level. $\beta$-glucans are taken as herbal medicines, to prevent and treat cancer, on human HIV/AIDS, and also used for common colds, influenza, and in many cases of alleged inflammation which is is consistent with hypoxia [24,25]. Several studies with mushroom biomass enhances the fact that besides the body producing several free radical quenching enzymes as its first line of defence, mushroom (Inonotus obliquus, Auricularia auricula and Poria cocos) nutrition supplies a significant exogenous source of these enzymes such as superoxide dismutase (SOD), catalase, and glutathione peroxidase [26]. Recently, our studies conducted with animal models showed favourable results on the use of biomass of mushroom Coriolus versicolor on Alzheimers Disease [27] and Ménier Disease.

People also use $\beta$-glucans orally to treat the skin with several dermatological hazards to maintain moisture and on sun radiation burns [28]. $\beta$-glucans of some mushrooms improve the host-mediated cancer immune response. Indeed, $\beta$-glucans, protein-bound polysaccharides, lignins, triterpenes, purines and polyphenols, not only modify the immune system but some of them are toxic to cancer cells by inducing necrosis and promoting apoptosis, while others subtly change their functioning [29].

Mushroom (Coriolus versicolor, Cordyceps sinensis, Grifola frondosa, Hericium erinaceus) $\beta$-glucans have been proposed to act as "biological response modifiers" (BRM, which include vaccines, monoclonal antibodies, cytokines, and adjuvants) based on its immunotherapy power, enhancing the body's own use of macrophages and T-lymphocytes, restoring the natural immune system rather than directly attacking the tumours [30]. The five key immune responses targeted by mushroom $\beta$-glucans involve the production of white blood cells, cellular mobilization, phagocytic capacity, production of reactive oxygen intermediates and help shift an overstimulated TH2 to a TH1 cell mediated immune response.

\section{Human Gut Microbiota}

The first line of defence against any pathogen invasion is the epithelial barrier. The vast army of commensal bacteria also prevent pathogen colonization and infection indirectly by enhancing host defence mechanisms being a vital component in maintaining human health. Some of the complex carbohydrates in plants cannot be digested by our bodies alone as humans do not secrete such specific enzymes. They have to be broken down by the gut microbiota, which produce enzymes to chop up the long chains and ferment them into short-chain fatty acids such as butyrate - which is made exclusively by bacteria acetate and propionate. These short-chain volatile fatty acids are beneficial to the body. Butyrate, for example, provides an energy source that the cells lining our intestines can directly access. It also controls the proliferation of cells in the intestine and is thought to possess anti-carcinogenic properties.

Bacteroides (B. ovatus, B. uniformis, B. capillosus), Prevotella, Ruminococcus, E. faecium, Streptococcus, and Clostridium strains seem to be responsible for degradation of mixed linked $\beta$-glucans in the small intestine and in the hind gut. Endogenous $\beta$-glucans show better prebiotic properties than exogenous $\beta$-glucans [31]. Bacterial derived $\beta$-glucans could be used as an effective compound inducing apoptosis in human colon cancer [32] and $\beta$-glucans from mushrooms on prostatic cancer [33].

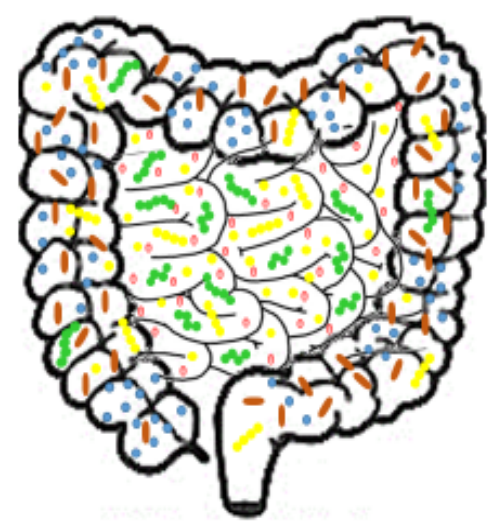

Proteobacteria
Lacto Acid Bacteria
(LAB); Firmicutes
Clostriadiales
Nutrients
Undigestible nutrients

Figure 2. Distribution of different bacteria through the gut

As our understanding of host gut microbiome increases, research interests in the fields of prebiotics, probiotics and synbiotics are growing rapidly. The major source of prebiotics is dietary fibre but one would have to eat a huge amount of these natural prebiotics, preferably raw, to gain any benefit from them. The majority of prebiotic supplements available are said to be all made of fibres, however, not all fibres are prebiotics. Besides, this statement is not correct since both copper and even sodium chloride may act as prebiotics. Very few investigations have been focused on non-digestible long chain complex carbohydrates for their potential as novel prebiotics. One of the reasons behind this is the unavailability of non-digestible polysaccharides that can fulfil the criteria as prebiotics. $\beta$-glucans that exist as nondigestible polysaccharides derived from mushrooms and other food sources have demonstrated not only health promoting effects, but also the potential as a novel source of prebiotics [34].

Mushrooms produce a vast set of extracellular carbohydrate-active enzymes and biological active molecules that are able to degrade very complex compounds such as hemicellulose and lignin. The variety of enzymes is dependent on the habitat and specific substrates so it differs among mushroom species and home ground. Fungi are good producers of cellulolytic extracellular enzymes and are widely used in many industrial processes. The diversity of cellulolytic-active enzymes is organized in different families of related amino acid sequences of the structurally related catalytic domains [35].

There is strong evidence suggesting that important antioxidant and cytoprotective enzymes are present in various edible fungi [36]. This points out the importance 
of a therapeutic strategy based on nutritional interventions with supplementation with biomass or extracts from mushroom to prevent and limit the deleterious consequences in many notable disorders associated with oxidative stress such as age-related diseases, high incidence of gastric cancer, coronary heart disease, asthma, inflammatory conditions, and neurodegenerative disorder.

Cell damage is induced by Reactive Oxygen Species (ROS) which can be either free radicals or molecules containing reactive oxygen atoms, i.e., hydrogen peroxide, superoxides, hydroxyl anions. Oxidant species produce free radicals or are activated chemically by them. Natural Killer cells (NK) are susceptible to ROS and lose their activity. High oxidation promotes DNA and tissue homeostasis and damage, aging pathologies, higher inflammation and gut disorders (Figure 3), diabetes and brain degeneration mainly in the most common chronic neurodegenerative Alzheirmers' and Parkinson's diseases.

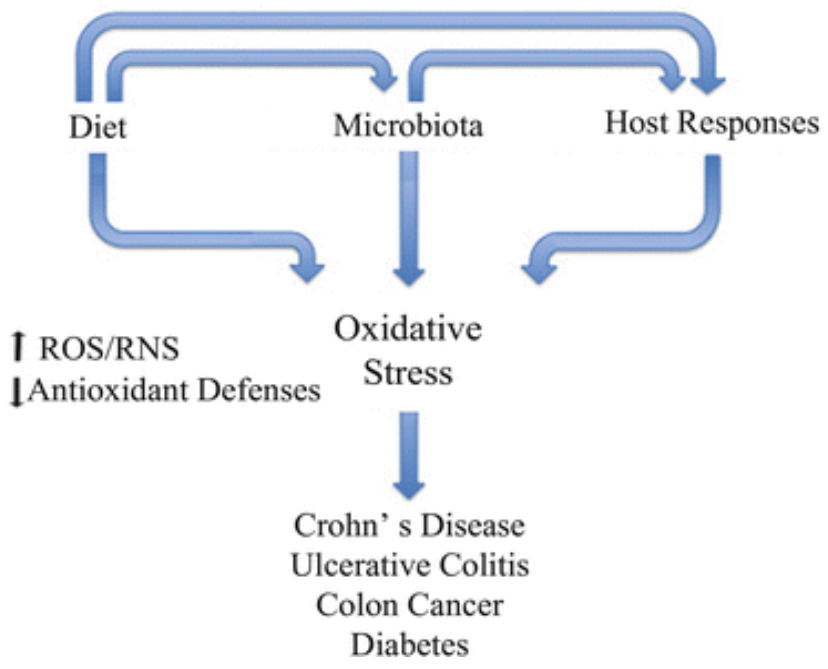

Figure 3. Relationship among diet, microbiota and host on oxidative stress

In general, oral administration of certain enzymes contribute to the reduction of chronic toxic overload in the organism and the optimisation of the following: a) balance $\mathrm{pH}$ levels: blood and extracellular matrix; b) removal of toxic substances; c) recovery of intestinal bacterial balance (biota balance); d) enhancement and balance of the immune system; e) improvement in cell metabolism [37].

There are numerous situations and conditions (Figure 4) related with oxidative stress [38].

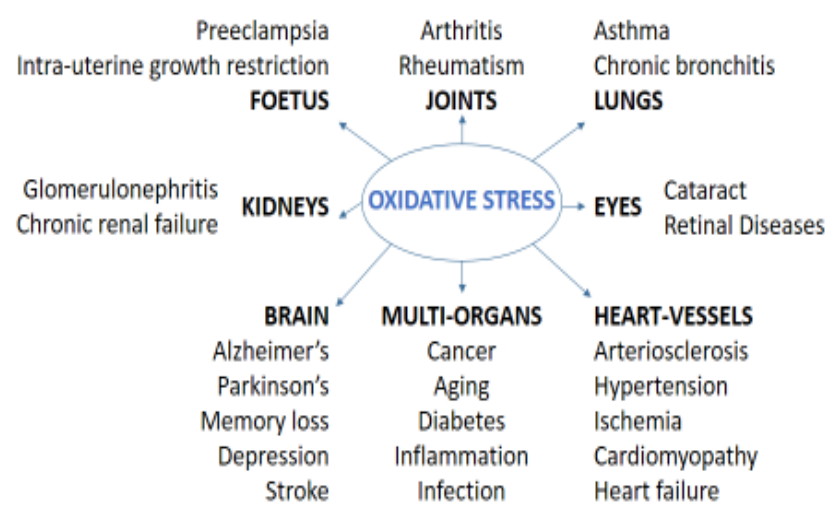

Figure 4. Oxidative stress-induced diseases in humans
Oxidative stress is a mechanism by which motor neuron death occurs and is characterized by elevated levels of intracellular reactive oxygen species (ROS), damage of TM cells, induction of apoptosis, and promotion of cellular aging. The human body is exposed to free radicals every day; they are produced in our bodies as part of normal functioning and may increase the risk of lifestyle and induce several disorders [39]. Free radicals and oxidants play a dual role as both toxic and beneficial compounds, since they can be either harmful or helpful to the body.

There is a range of enzymes related to oxidative stress which are involved in generating deleterious free radical species while others have antioxidant properties. The human body has several mechanisms to counteract oxidative stress by producing antioxidants, which are either naturally produced in situ, or externally supplied through foods and/or supplements [40].

\section{Immuno-activating and protective effects of $\beta-1,3 / 1,6$-glucans}

Over 100,000 phytochemicals have been identified. Phytochemicals which are present in the vegetable/plant foodstuffs are natural, non-nutritive elements which act as protective agents for the plants and they are recognized to possess a number of beneficial effects on human health. Scientific evidence showed that mushrooms derived compounds are valuable sources as therapeutics and immunomodulators. For example, phytochemical screening of edible white oyster mushroom, Pleurotus florida, reveals the presence of phenols, flavonoids, alkaloids, terpenoids, tannins, saponins and glycosides in mushroom dried powder and in diverse enzyme content of several mushrooms [41].

The classic model of mode of action of $\beta$-glucans, as soluble (soluble viscous fibres delay gastric emptying time and slow glucose absorption) and fermentable fibres by microbiota, is that they are recognized by the immune system as an invasive organism since they are only present in plants and not in the human or animal bodies, hence activating the body's immune defences as if exposed to infection. The mushroom mycelium is the network of fungal threads in soil that acts as interfaces between plant roots, bacteria and nutrients to plants, rescuing ecosystems. The proprietary biomass forms of mushroom preparations, contrary to industrial extract forms containing just polysaccharide peptides, includes the mycelium therefore this type of dietary supplements may have the ability to defend from infectious diseases not only through direct anti-microbial activity but also from the production of our immune cells [25,42].

Nevertheless, the immune system is not a single entity and is activated through many different factors and hence by many of mushroom components being $\beta$-glucans the element better studied. Other constituents work synergistically to optimize lifestyle and promote health [43]. Presently our team is investigating the role of metalloids as factors that may also trigger health benefits through different diet sources including mushrooms.

The yield of the immune system cells can be stimulated however this is quite complex since there are so countless 
different kinds of cells in the immune system that respond differently to numerous types of microbes present in the gut. There are two main classes of T-lymphocytes: a) Cytotoxic T cells (CTL or Tc cell); b) Helper T cells (Th cells). Cytotoxic T cells usually target and illicit immune effector response against the self-altered cells like virus or bacterial infected cells or cancerous cells. CTL kill the host cell in a directly in that they trigger a programmed cell death, apoptosis being the best-characterised and most evolutionary conserved form of programmed cell death. The Th cells, stimulate the immune reaction by expressing CD4 and CD8 cells, and activating NK-natural killer cells, macrophages and B cells (Figure 5) [44].

Different types of mushrooms produce different strains of $\beta$-glucans and different concentrations. Reishi, Cordyceps, and other medicinal mushrooms each offer different health benefits. And other unique active substances in the different mushrooms trigger unique receptors of the immune system, or target receptors on microbes or malignant cells (Figure 5). The body may benefit from a lot of different active substances derived from unique mushroom sources to lift the immune system relatively quickly (Figure 6). The metabolites in mushrooms create reactions from the flora in our guts in preserving the probiotics and modulating intercellular signalling [45].

The more interesting molecules are the glycoproteins PSK and PSP profoundly studied by Asian scientists as extracts from mushrooms. The glycoprotein with 2 moieties of polysaccharide and polypeptide create some interesting cellular actions on cell membrane. Eating mushrooms everyday may affect health not only from the standpoint of nutrition, but also from helping many of our physiological functions such as immunity, digestion, and neutralizing fats. However, there is a huge difference between fresh mushrooms, their biomass or extracts.

Once we venture into the healing arena, we start to conform to the classic pharmacology premises of single molecule/single indication paradigm. There is a wrong urge to refine a natural product to make it look more like a drug. However, dietary supplements have legal status similar to that of common foodstuffs while drugs are usually mono-molecular substances and deeply scrutinized by recognised authorities.

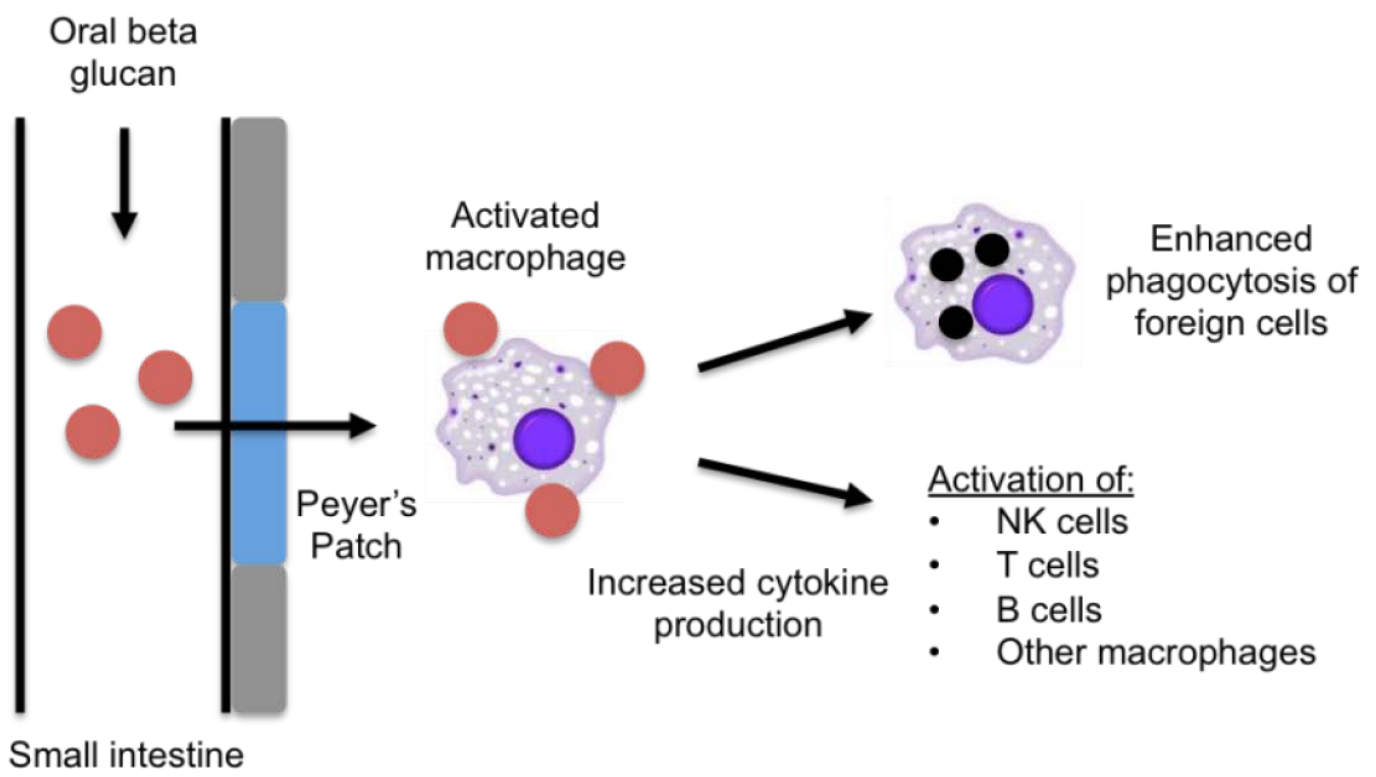

Figure 5. $\beta$-glucans and activation of the immune system.

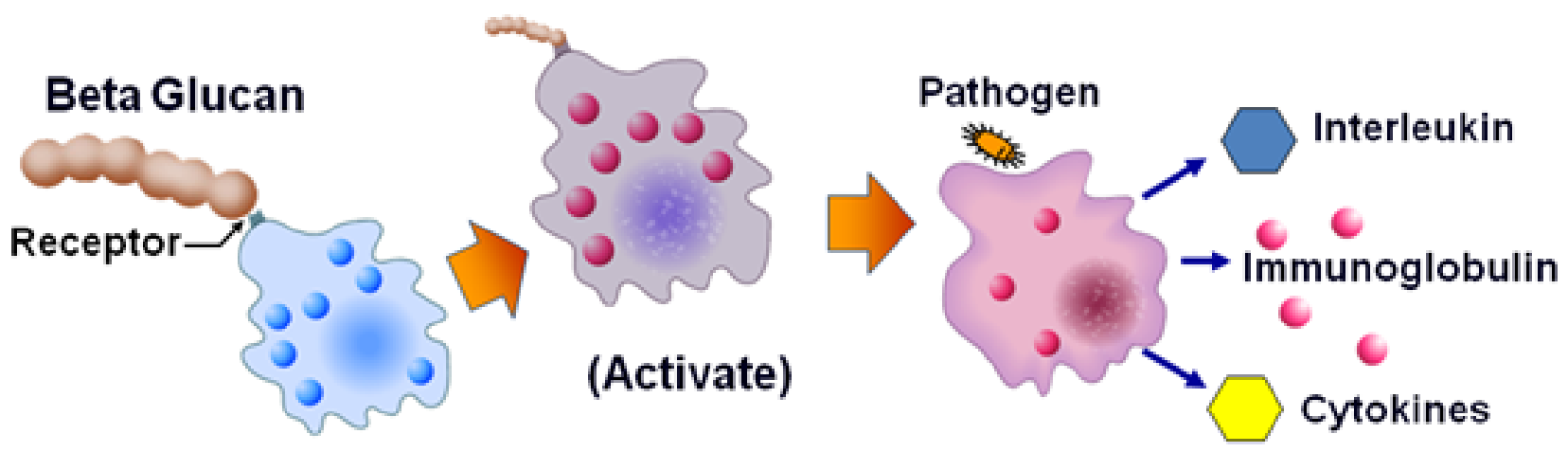

\section{Macrophage activation via cell surface receptor}

Figure 6. The role of $\beta$-glucans on the immune system 


\section{Conclusion}

The human gut is a dynamic environment where eukaryotic and a multitude of prokaryotic cells establish a complex network of communication that ultimately benefits both the "microflora party" and the human host. There must be in the future the possibility of identifying host factors implicated in the disruption of the intestinal microbiota and associated pathologic consequences.

Although the overwhelming majority of genes (99.1\%) examined by metagenomic sequencing of the intestinal tract are bacterial in origin, it will be important to have a complete characterization of the enteric microbial communities, i.e., bacteria, viruses, fungi, and archaea, which presence surely influence specific host response and intestinal homeostasis. Studies should proceed regarding the enteric microbiota community as a whole as they represent a very complex relationship.

Consequently, a targeted manipulation of microbial activities when feeding mushrooms or their preparations may represent a better approach to understand their impact on disease treatment and management.

Mushrooms have been used for several thousands of years for medicinal purposes, however their role on the regulation of gut microbiota is a new field and has not been sufficiently studied among the 2,000 species identified as edible.

\section{References}

[1] Ursell LK, Clemente JC, Rideout JR, Gevers D, Caporaso JG, Knight R. (2012) The interpersonal and intrapersonal diversity of human-associated microbiota in key body sites. J Allergy Clin Immunol. 129: 1204-1208.

[2] Lurie-Weinberger MN, Gophna U (2015) Archaea in and on the Human Body: Health Implications and Future Directions. PLoS Pathog 11(6): e1004833.

[3] Fallon PG, Alcami A. (2006). Pathogen-derived immunomodulatory molecules: future immunotherapeutics? Trends Immunol. 2006 Oct; 27(10):470-6. Epub 2006 Aug 21.

[4] Xu J, Gordon JI. (2003). Honor thy symbionts. Proc. Natl. Acad. Sci. USA 100: 10452-10459.

[5] Surana NK, Kasper DL. (2012). The yin yang of bacterial polysaccharides: Lessons learned from $B$. fragilis PSA. Immunol Rev. 2012 Jan; 245(1): 13-26.

[6] Gershon M. (1998). The Second Brain: The Scientific Basis of Gut Instinct and a Groundbreaking New Understanding of Nervous Disorders of the Stomach and Intestines. Publisher: Harper; 1 edition (October 7, 1998).

[7] Furness JB. (2008). The Enteric Nervous System. John Wiley \& Sons. pp. 35-38.

[8] Miron J, Ben-Ghedalia D, Morrison M. (2001). Invited review: adhesion mechanisms of rumen cellulolytic bacteria. J Dairy Sci. 2001 Jun; 84(6): 1294-309.

[9] Arumugam M, Raes J, Pelletier E, Le Paslier D, Yamada T, Mende DR, Fernandes GR, Tap J, Bruls T, Batto JM, Bertalan M, Borruel N, Casellas F, Fernandez L, Gautier L, Hansen T, Hattori M, Hayashi T, Kleerebezem M, Kurokawa K, Leclerc M, Levenez F, Manichanh C, Nielsen HB, Nielsen T, Pons N, Poulain J, Qin J, Sicheritz-Ponten T, Tims S, Torrents D, Ugarte E, Zoetendal EG Wang J, Guarner F, Pedersen O, de Vos WM, Brunak S, Doré J; MetaHIT Consortium, Antolín M, Artiguenave F, Blottiere HM, Almeida M, Brechot C, Cara C, Chervaux C, Cultrone A, Delorme C, Denariaz G, Dervyn R, Foerstner KU, Friss C, van de Guchte M, Guedon E, Haimet F, Huber W, van Hylckama-Vlieg J, Jamet A, Juste C, Kaci G, Knol J, Lakhdari O, Layec S, Le Roux K, Maguin E, Mérieux A, Melo Minardi R, M'rini C, Muller J, Oozeer R, Parkhill J, Renault P, Rescigno M, Sanchez N, Sunagawa S, Torrejon A, Turner K, Vandemeulebrouck G, Varela
E, Winogradsky Y, Zeller G, Weissenbach J, Ehrlich SD, Bork P. (2011) Enterotypes of the human gut microbiome. Nature 473 174-180.

[10] Yazawa S, Takahashi R, Yokobori T, Sano R, Mogi A, Saniabadi AR, Kuwano H, Asao T. (2016). Fucosylated Glycans in $\alpha 1$-Acid Glycoprotein for Monitoring Treatment Outcomes and Prognosis of Cancer Patients. Singh PK, ed. PLoS ONE. 2016; 11(6): e0156277.

[11] Grass J, Pabst M, Kolarich D, Pöltl G, Léonard R, Brecker L, Altmann F. (2011). Discovery and Structural Characterization of Fucosylated Oligomannosidic N-Glycans in Mushrooms. February 25, 2011. The Journal of Biological Chemistry. 286, 5977-5984.

[12] Frey-Klett P, Burlinson P, Deveau A, Barret M, Tarkka M, Sarniguet A. (2011). Bacterial-Fungal Interactions: Hyphens between Agricultural, Clinical, Environmental, and Food Microbiologists. Microbiol. Mol. Biol. Rev. December 2011 vol. 75 no. 4 583-609.

[13] Tarkka MT, Sarniguet A, Frey-Klett P. (2009). Inter-kingdom encounters: recent advances in molecular bacterium-fungus interactions. Curr. Genet. 55: 233-243.

[14] Wu GD, Chen J, Hoffmann C, Bittinger K, Chen YY, Keilbaugh SA, Bewtra M, Knights D, Walters WA, Knight R, Sinha R, Gilroy E, Gupta K, Baldassano R, Nessel L, Li H, Bushman FD, Lewis JD (October 7, 2011). "Linking long-term dietary patterns with gut microbial enterotypes". Science. 334 (6052): 105-8.

[15] Zoetendal EG, Rajilic-Stojanovic M, de Vos WM. (2008). Highthroughput diversity and functionality analysis of the gastrointestinal tract microbiota. Gut 57, 1605-1615.

[16] Salvucci E. (2014). Microbiome, holobiont and the net of life. Critical Reviews in Microbiology: 1-10.

[17] Littman DR, Pamer EG. (2011). Role of the commensal microbiota in normal and pathogenic host immune responses. Cell Host Microbe. 2011 Oct 20; 10(4): 311-23.

[18] Arora NK, Kim MJ, Kang SC, Maheshwari DK. (2007). Role of chitinase and beta-1,3-glucanase activities produced by a fluorescent pseudomonad and in vitro inhibition of Phytophthora capsici and Rhizoctonia solani. Can. J. Microbiol. 53: 207-212.

[19] Inman M (2011) How Bacteria Turn Fiber into Food. PLoS Biol 9 (12): e1001227.

[20] Mazmanian SK, Round JL, Kasper DL. (2008). A microbial symbiosis factor prevents intestinal inflammatory disease. Nature. 2008 May 29; 453(7195): 620-5.

[21] The Human Microbiome Project Consortium. Structure, Function and Diversity of the Healthy Human Microbiome. Nature. 2012 Jun 14; 486(7402): 207-214. Published online 2012 Jun 13.

[22] Rea K, G.Dinan TG, Cryan JF. (2016). The microbiome: A key regulator of stress and neuroinflammation. Neurobiology of Stress. Volume 4, October 2016, Pages 23-33.

[23] Oriach CS, Robertson RC, Stanton C, Cryan JF, Dinan TG. (2016) Food for thought: The role of nutrition in the microbiota-gut-brain axis. Clinical Nutrition Experimental. Volume 6, April 2016, Pages 25-38.

[24] Jayachandran M, Xiao J, Xu B. A Critical Review on Health Promoting Benefits of Edible Mushrooms through Gut Microbiota. International Journal of Molecular Sciences. 2017; 18(9): 1934.

[25] Ferrão J, Bell V, Calabrese V, Pimentel L, Pintado M, Fernandes TH. Impact of Mushroom Nutrition on Microbiota and Potential for Preventative Health. Journal of Food and Nutrition Research. Vol. 5, No. 4, 2017, pp 226-233.

[26] Karmali A. (2017). Comparative Enzyme Analysis of Inonotus obliquus (Chaga), Auricularia auricula and Poria cocos. Clin J Mycol. 2017; 5: 2-4.

[27] Trovato A, Pennisi M, Crupi R, Di Paola R, Alario A, Modafferi S Di Rosa G, Fernandes T, Signorile A, Maiolino L, Cuzzocrea S, Calabrese V. (2017). Neuroinflammation and Mitochondrial Dysfunction in the Pathogenesis of Alzheimer's Disease: Modulation by Coriolus Versicolor (Yun-Zhi) Nutritional Mushroom. J Neurol Neuromed (2017) 2(1): 19-28.

[28] Pillai R, Redmond M, Röding J. (2005). Anti-Wrinkle Therapy: Significant New Findings in the Non-Invasive Cosmetic Treatment of Skin Wrinkles with Beta-Glucan. The Global Publication of the International Federation of Societies of Cosmetic Chemists. Vol.8. No 1 . http://www.nononsensecosmethic.org/wpcontent/uploads/2015/02/00463528995290fd55000000.pdf.

[29] Yuen JWM, Gohel MDI. (2005). Anticancer Effects of Ganoderma lucidum: A Review of Scientific Evidence. Journal Nutrition and Cancer. Volume 53, 2005 - Issue 1. 
[30] Ng TB (1998). A review of research on the protein-bound polysaccharide (polysaccharopeptide, PSP) from the mushroom Coriolus versicolor (Basidiomycetes: Polyporaceae). Gen Pharmacol 30 (1): 1-4.

[31] Beckmann L, Simon O, Vahjen W. (2006). Isolation and identification of mixed linked beta -glucan degrading bacteria in the intestine of broiler chickens and partial characterization of respective 1,3-1,4-beta -glucanase activities. J Basic Microbiol. 2006; 46(3): 175-85.

[32] Kim MJ, Hong SY, Kim SK, Cheong C, Park HJ, Chun HK, Jang KH, Yoon BD, Kim CH, Kang SA. (2009). $\beta$-glucan enhanced apoptosis in human colon cancer cells SNU-C4. Nutr Res Pract. 2009 Fall; 3(3): 180-4.

[33] Fullerton SA, Samadi AA, Tortorelis DG, Choudhury MS, Mallouh C, Tazaki H, Konno S. (2000). Induction of apoptosis in human prostatic cancer cells with beta-glucan (Maitake mushroom polysaccharide). Mol Urol. 2000. Spring; 4(1): 7-13. PMID: 10851301.

[34] Lam, K-L, Cheung P. (2013). Non-digestible long chain betaglucans as novel prebiotics. Bioactive Carbohydrates and Dietary Fibre. 2. 45-64.

[35] Battaglia E, Benoit I, Brink J, Wiebenga A, Coutinho PM, Henrissat B, Vries RP. (2011). Carbohydrate-active enzymes from the zygomycete fungus Rhizopus oryzae: a highly specialized approach to carbohydrate degradation depicted at genome level. BMC Genomics. 2011; 12: 38.

[36] van den Brink J, de Vries RP. (2011). Fungal enzyme sets for plant polysaccharide degradation. Appl Microbiol Biotechnol. 2011 Sep; 91(6): 1477-1492. Published online 2011 Jul 23.

[37] Barros AB, Bell V, Ferrão J, Calabrese V, Fernandes TH. (2016). Mushroom Biomass: Some Clinical Implications of $\beta$-Glucans and Enzymes. Curr Res Nutr Food Sci 2016; 4 (Special Issue Confernce October 2016).
[38] Chiurchiù V, Maccarrone M. (2011). Chronic inflammatory disorders and their redox control: from molecular mechanisms to therapeutic opportunities. Antioxid Redox Signal. 2011 Nov 1; 15(9): 2605-41.

[39] Nita M, Grzybowski A. The Role of the Reactive Oxygen Species and Oxidative Stress in the Pathomechanism of the Age-Related Ocular Diseases and Other Pathologies of the Anterior and Posterior Eye Segments in Adults. Oxidative Medicine and Cellular Longevity. 2016; 2016: 3164734.

[40] Ribeiro B, Rangel J, Valenta OP, Baptista P, Seabra RM, Andrade PB. (2006). Contents of carboxylic acids and two phenolics and antioxidant activity of dried portuguese wild edible mushrooms. $J$. Agric. Food Chem. 54: 85308537.

[41] Prabu M. (2015). Evaluation of phytochemicals and in vitro antiinflammatory, anti-diabetic activity of the white oyster mushroom, Pleurotus florida.

https://www.researchgate.net/publication/280254307.

[42] Sommer F., Bäckhed F. The gut microbiota-masters of host development and physiology. Nat Rev Microbiol. 2013; 11: 227-238.

[43] Marshall NB, Swain SL. (2011). Cytotoxic CD4 T Cells in Antiviral Immunity. Biomed Biotechnol. 954602. Published online 2011 Nov 22

[44] Ma L, Chen H, Zhu W, Wang Z. (2013). "Effect of different drying methods on physicochemical properties and antioxidant activities of polysaccharides extracted from mushroom Inonotus obliquus." Food Research International. 50: 633-640.

[45] Chang C-J, Lin C-S, Lu C-C, Martel J, Ko Y-F, Ojcius DM, Tseng S-F, Wu T-R, Chen, John D. Young Y-YM, Lai H-C. (2015). Ganoderma lucidum reduces obesity in mice by modulating the composition of the gut microbiota. Nature Communications. Vol.6. Article number: 7489 (2015). 